\title{
Nonlinear and fault-tolerant flight control using multivariate splines
}

\author{
H.J. Tol, C.C. de Visser, E. van Kampen and Q.P. Chu
}

\begin{abstract}
This paper presents a study on fault tolerant flight control of a high performance aircraft using multivariate splines. The controller is implemented by making use of spline model based adaptive nonlinear dynamic inversion (NDI). This method, indicated as SANDI, combines NDI control with nonlinear control allocation based on an onboard aerodynamic spline model and a real-time identification routine. The controller is tested for an aileron hardover failure and structural damages which change the global aerodynamic properties of the aircraft. It is shown that the controller can quickly tune itself in failure conditions without the need of failure detection and monitoring algorithms. Instead, self-tuning innovation based forgetting is applied to reconfigure the onboard aerodynamic model. The controller is able to tune itself each time a model error is detected and does not require any external triggers for re-identification. Multivariate splines have a high local approximation power and are able to accurately model nonlinear aerodynamics over the entire flight envelope of an aircraft. As a result the identification routine gives a robust adaption of the aerodynamic model in case of a failure.
\end{abstract}

H.J. Tol

Delft university of technology, 2600 GB Delft, e-mail: h.j.tol@tudelft.nl

C.C. de Visser

Delft university of technology, 2600 GB Delft, e-mail: c.c.devisser@tudelft.nl

E. van Kampen

Delft university of technology, 2600 GB Delft, e-mail: E.vanKampen@tudelft.nl

Q.P. Chu

Delft university of technology, 2600 GB Delft, e-mail: q.p.chu@tudelft.nl 


\section{Glossary}

$A_{x}, A_{y}, A_{x}$ specific forces along the body $\mathrm{X} / \mathrm{Y} / \mathrm{Z}$ axis $\left[\mathrm{m} / \mathrm{s}^{2}\right]$

$C_{l}, C_{m}, C_{n}$ aerodynamic moment coefficients around the body $\mathrm{X} / \mathrm{Y} / \mathrm{Z}$ axis

$I$ inertia matrix

$S$ wing area $\left[m^{2}\right]$

$V$ airspeed $[\mathrm{m} / \mathrm{s}]$

$b$ wing span $[\mathrm{m}]$

$\bar{c}$ mean aerodynamic chord $[\mathrm{m}]$

$p, q, r$ roll, pitch and yaw rate around the body $\mathrm{X} / \mathrm{Y} / \mathrm{Z}$ axis $[\mathrm{rad} / \mathrm{s}]$

$p_{s}$ static pressure [Pa]

$\bar{q}$ dynamic pressure $[\mathrm{Pa}]$

$u, v, w$ velocity components along the body $\mathrm{X} / \mathrm{Y} / \mathrm{Z}$ axis $[\mathrm{m} / \mathrm{s}]$

$\mathbf{u}$ input vector

x state vector

$\alpha, \beta$ angle of attack and sideslip angle [rad]

$\phi, \theta, \psi$ roll, pitch and yaw angle [rad]

$\delta_{e}, \delta_{a}, \delta_{r}, \delta_{l e f}$ elevator, aileron, rudder and leading edge flap control surface deflection [rad]

$\varepsilon$ model error

$\lambda$ forgetting factor

$\rho$ air density $\left[\mathrm{kg} / \mathrm{m}^{3}\right]$

$\tau$ virtual input

$v$ virtual input

$\Sigma_{0}$ filter information content

$\sigma$ time constant 


\section{Introduction}

This paper presents a study on fault tolerant flight control using multivariate splines. Self-tuning adaptive control is applied in which a real time identification routine is used to supply updated information to the control system in case of a failure. The controller is based on the nonlinear dynamic inversion principle in which the aerodynamic model is used to linearize the system after which single linear controllers can be used to control the system [1-3]. Currently, most adaptive NDI controllers use either polynomial structures [4-6] or neural networks [7,8] for their internal model.

In this study multivariate simplex splines are used for the internal model. A multivariate spline is a piecewise defined polynomial function in which each basis polynomial is defined on a simplex [9, pp. 18-25]. Any number of basis polynomials can be combined with predefined continuity by combining simplices into a geometric structure called a triangulation. The approximation power of simplex spline functions is therefore is not only proportional to the polynomial degree, but also to the number and density of the polynomial pieces. Multivariate splines are linear in the parameters and are easily integrated into standard and recursive parameter estimation routines $[10,11]$.

Recently a new framework for spline model based NDI flight control is introduced in [12]. It is shown that the use of splines significantly improves the performance of NDI based control systems compared with ordinary polynomial based NDI. The controller from [12] is now augmented with a real time identification and applied for fault-tolerant flight control. First the aircraft model and the failure scenarios are discussed in Sec. 2. In Sec. 3 the fault tolerant control method is presented and in Sec. 4 the controller is evaluated.

\section{Aircraft Model and Failure Cases}

The aircraft to be controlled in this study is a model of the F-16 fighter aircraft from NASA, which is based on a set of data tables based on wind-tunnel measurements [13]. The model has the traditional aerodynamic control surfaces: elevator, ailerons and rudder for pitch, roll and yaw control. In addition, the leading edge flap is scheduled with angle of attack and $\frac{\bar{q}}{p_{s}}$ to optimize performance [13]. Models for the actuators are included in the form of first order lags:

$$
\dot{u}=\frac{1}{\sigma}\left(u_{c o m}-u\right)
$$

In which the commanded input is bounded by $u_{\text {min }} \leq u_{\text {com }} \leq u_{\text {max }}$ and the deflection rate is bounded by $|\dot{u}| \leq \dot{u}_{\text {lim }}$. The time constants $\sigma$ and actuator limits are listed in table 1 which are taken from [13] and [14, pp.633-664]. For simulating the response and for flight control design the flat earth, body axis six degree of freedom equations 
of motion are used [14, pp. 107-116]. All simulations are performed in a deterministic environment. No external disturbances like wind gusts are added to the models and the sensor information is considered to be noise free. Stochastic adaptive control is reserved for future studies. Two failure cases are considered:

Table 1 Actuator model

\begin{tabular}{lccc}
\hline \hline & \multicolumn{3}{c}{ deflection limit rate limit time constant } \\
\hline Elevator & $\pm 25.0^{\circ}$ & $60^{\circ} / \mathrm{s}$ & $0.0495 \mathrm{~s} \mathrm{lag}$ \\
Ailerons & $\pm 21.5^{\circ}$ & $80^{\circ} / \mathrm{s}$ & $0.0495 \mathrm{~s} \mathrm{lag}$ \\
Rudder & $\pm 30.0^{\circ}$ & $120^{\circ} / \mathrm{s}$ & $0.0495 \mathrm{~s} \mathrm{lag}$ \\
leading edge flap & $0^{\circ}-25^{\circ}$ & $25^{\circ} / \mathrm{s}$ & $0.136 \mathrm{~s} \mathrm{lag}$ \\
\hline \hline
\end{tabular}

\section{Aileron hardover}

The first failure scenario is a aileron hardover in which the right aileron gets stuck in an offset position. The failure scenario is described by:

$$
\delta_{a_{a c t u a l}}=\delta_{a_{\text {left }}}+\delta_{a_{\text {right }}}=\frac{1}{2} \delta_{a_{\text {command }}}+\delta_{a_{\text {offset }}}
$$

The offset position is chosen as $\delta_{a_{\text {offset }}}=7^{\circ}$. Due to the hardover failure the aileron channel will become less effective. Furthermore, the aircraft will get a constant nominal rolling moment which needs to be compensated by the left aileron.

\section{Structural damage}

The second failure case considered is a structural damage. It is assumed the structural damage changes the global aerodynamic properties of the aircraft. Two types of aerodynamic changes are considered based on earlier work by Choi et al [15]:

1. Magnitude scaling: In this case the coefficients in failure conditions are obtained by scaling the magnitude of the nominal aerodynamic coefficients from the lookup table:

$$
C_{a c t}(\mathbf{x})=\left[1+a_{\text {mag }}\right] C_{\text {nom }}(\mathbf{x})
$$

2. Variable scaling: In this case the coefficients in failure conditions are obtained by scaling the independent variable of the aerodynamic coefficient from the look-up table:

$$
C_{\text {unc }}(\mathbf{x})=C_{a c t}\left(\left[1+a_{\text {var }}\right] \mathbf{x}\right)
$$

A combined magnitude scaling and variable scaling with $a_{m a g}=a_{v a r}=-0.3$ applied to all coefficients from the lookup table. In addition the center of gravity is shifted $10 \%$ rearward to make the actual aircraft model less stable. Fig. 1 visualizes 
the uncertainties for the pitch moment coefficients. It can be observed that these uncertainties have a nonlinear effect on the model especially in the high angle of attack operating region.
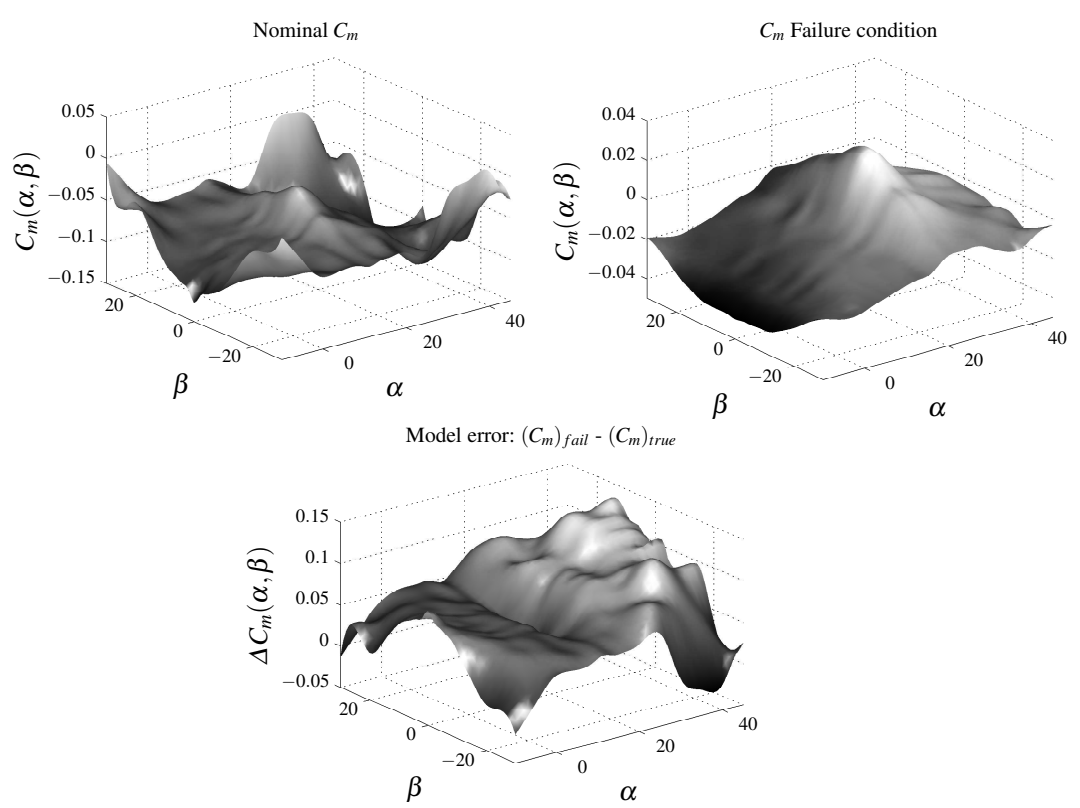

Fig. $1 C_{m}$ surface plots generated from data tables without uncertainties (top left) and with uncertainties (top right) $\left(\tilde{q}=0.0015, \delta_{e}=5^{\circ}, \delta_{l e f}=10^{\circ}\right)$

\section{Fault Tolerant Flight Control Method}

The fault tolerant control method used in this study is spline model based adaptive NDI (SANDI). A structure for spline based NDI control was first introduced in [9] which combines NDI control with nonlinear control allocation based on the onboard spline model. This structure is now augmented with a self tuning online estimator for simplex splines and applied to control the F-16 aircraft in failure conditions. The control diagram is shown in Fig. 2. The architecture has three major assemblies: 1) NDI control augmented with 2) control allocation based on the onboard aerodynamic spline model and 3) a real-time model identification routine. The aim of this setup is to update an a-priory aerodynamic model online in case of a failure with adaptive simplex splines to eliminate model errors within the NDI control system. In [12] the F-16 aerodynamic model is identified with multivariate splines using flight testing based system identification techniques from $[10,16,17]$. This is an accurate global model and is used as the a-priori nominal onboard aerodynamic 
model in this study. Refer to [12] for the complete structure, the accuracy and error bounds of the model.

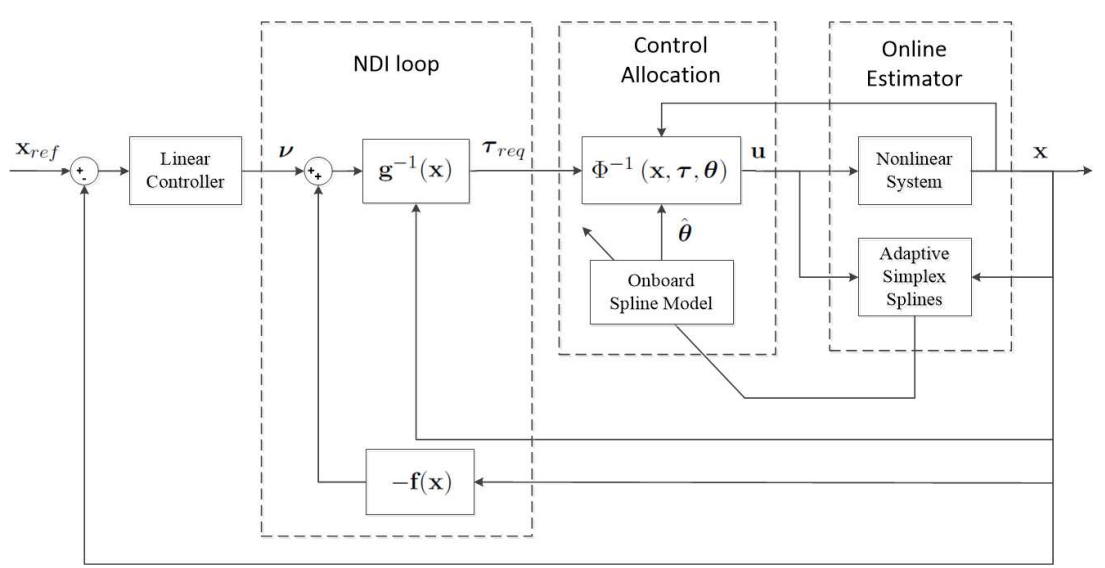

Fig. 2 Overview of the spline based adaptive control setup

Consider the aircraft state equations in the input affine form:

$$
\begin{aligned}
\dot{\mathbf{x}} & =\mathbf{f}(\mathbf{x})+g(\mathbf{x}) \tau \\
\tau & =\Phi(\mathbf{x}, \mathbf{u}, \Theta) .
\end{aligned}
$$

with $\mathbf{x} \in \mathbb{R}^{n}$ the state vector, $\mathbf{u} \in \mathbb{R}^{m}$ the control input vector, $\tau \in \mathbb{R}^{l}$ representing the aerodynamics assumed to be a nonlinear function of the aircraft state and control input, and with $\Theta \in \mathbb{R}^{r}$ the parameter vector to be updated in case of a failure. With NDI the system is linearized by solving for the input $\tau$ by introducing an outer loop control input $v$ :

$$
\tau_{r e q}=g^{-1}(\mathbf{x})(v-\mathbf{f}(\mathbf{x}))
$$

Which results in a closed-loop system with a decoupled linear input-output relation:

$$
\dot{\mathbf{x}}=v
$$

Eq. (6) represents the aerodynamic model and maps the physical control inputs to the virtual controls, i.e. to the forces and moments of the aircraft:

$$
\tau=\Phi(\mathbf{x}, \mathbf{u}, \Theta): \mathbb{R}^{m} \rightarrow \mathbb{R}^{l}
$$

The spline model for $\tau$ is non-affine in the controls. Furthermore, the basis polynomials of the simplex splines are defined locally on each simplex in terms of barycentric coordinates instead of globally in terms of Cartesian coordinates [9, pp.18-25]. Therefore the NDI control structure is augmented with a separate control allocation module that determines the physical control inputs for a required demand $\tau_{\text {req }}$ 
from the NDI control law Eq. (7). For flight control purposes the required moment coefficients have to be translated into control surface deflections based on the onboard spline model. Note that by separating the NDI loop from the control allocation task the need to reconfigure the NDI control laws to accommodate for the failure is avoided. Instead, reconfiguration is completely encapsulated within the control allocation module. The control allocation problem is solved by minimizing the least square errors with respect to the control input $\mathbf{u}$ :

$$
\min _{\underline{\mathbf{u}} \leq \mathbf{u} \leq \overline{\mathbf{u}}} \mathscr{J}=\left\|s(\mathbf{x}, \mathbf{u}, \Theta)-\tau_{r e q}\right\|_{2}^{2}=\sum_{i=1}^{N}\left(s_{i}(\mathbf{x}, \mathbf{u}, \Theta)-\tau_{r e q_{i}}\right)^{2}
$$

The derivation of the solution for Eq. (10) is not trivial, refer to [12] for the analytic derivation and solution for this optimization problem.

\subsection{Adaptive simplex splines}

A real time identification algorithm is used to reconfigure the aerodynamic model in failure conditions. The algorithm combines a recursive squares least estimator for multivariate splines [11] with an innovation based self-tuning forgetting factor [18], see Fig. 3.

A disadvantage of applying real time identification routines for fault-tolerant control is that the parameter vector converges quickly after which new data has no or little impact on the parameter estimate; the estimator has saturated. However, a fault-tolerant controller must be able to identify time-varying models in failure conditions. A frequently used approach to ensure adaptivity of the identification routine is to incorporate a trigger for re-identification of the aerodynamic model, see e.g $[4,5]$. A disadvantage of this approach is that it requires monitoring algorithms to identify and isolate the failure and it requires significant off-line analysis in order to define robust reset triggers.

In this study adaptivity is ensured by applying a variable forgetting factor proposed by Fortescue in [18] in the RLS algorithm which is tuned based on the RLS innovation:

$$
\lambda(t)=1-[1-X(t) L(t)] \varepsilon^{2}(t) / \Sigma_{0}
$$

with $X$ the row regression matrix for a new observation, $L$ the adaptation gain of the filter and, $\varepsilon$ the innovation and with $\Sigma_{0}$ a measure of the filter's information content. By keeping the information content constant it is ensured that the estimation is always based on the same amount of information. The amount of forgetting at each time step corresponds to the amount of new information in the latest measurement. In nominal flight conditions the estimation error will be small. Either the system has not been excited, there has been an excitation with the correct set of parameter values, or the estimator is sensitive enough to reduce the estimation error. In all these cases the forgetting factor is close to one. However in failure conditions when the error becomes larger the sensitivity of the estimator is increased and the estimator 
starts discounting past data in favor of more recent data. $\Sigma_{0}$ controls the speed of the adaptation and determines the sensitivity of the system. A small value for $\Sigma_{0}$ gives a large covariance matrix and a sensitive system; a larger value will give a less sensitive system and slower adaptation. However choosing a larger $\Sigma_{0}$ increases the robustness of the parameter estimate.

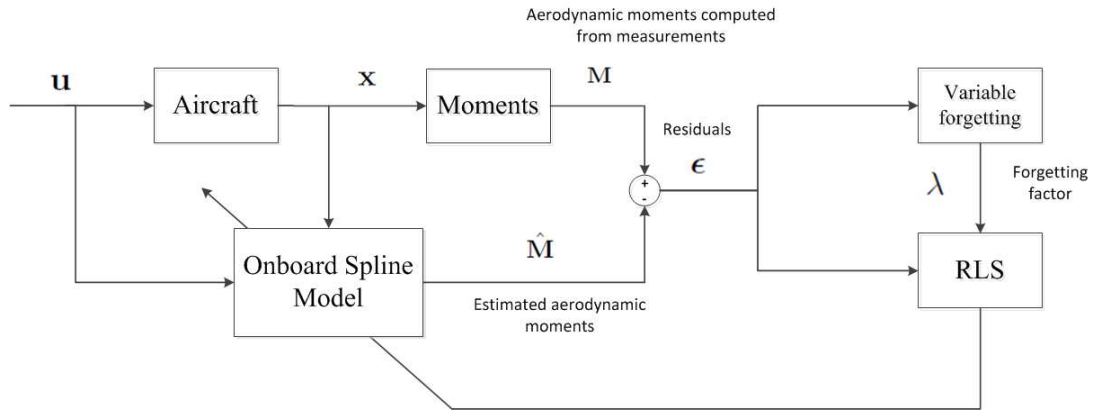

Fig. 3 Adaptive splines

\subsection{NDI flight control system}

An NDI flight control system is implemented using a two loop time-scale separated design [1], an inner rate control loop and an outer angle of sideslip control loop. This control setup is frequently used for manual flight control [5,19-21] and is shown in Fig. 4. First order lag prefilters $\left(H_{p f}=\frac{1}{\sigma s+1}\right)$ are added to ensure comfortable aircraft response and to avoid unachievable commands due to the actuator rate limits. Only proportional control is used for the sideslip feedback and pitch rate feedback.

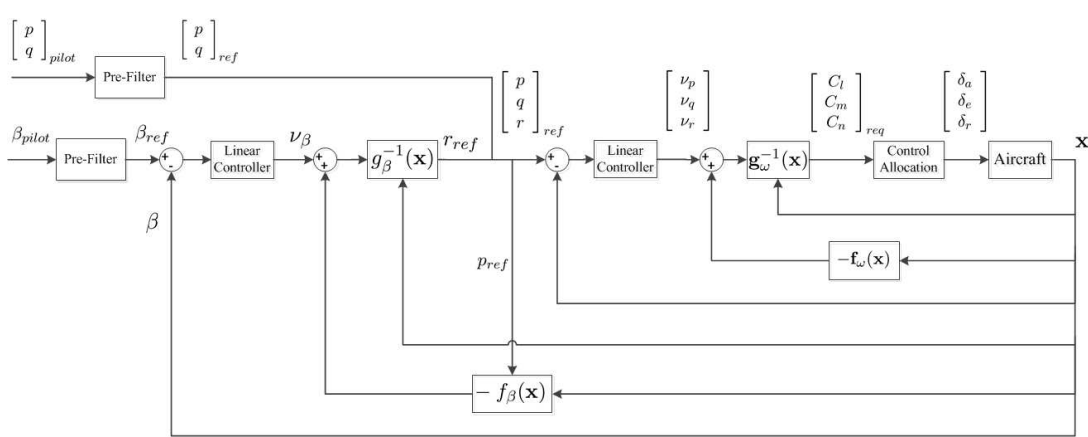

Fig. 4 Control setup. An angular rate inner NDI loop combined with a sideslip angle outer NDI loop 
In the inner loop the system is influenced by commanding the moments coefficients of the aircraft. The inner loop quantities are the body angular rates:

$$
\left[\begin{array}{c}
\dot{p} \\
\dot{q} \\
\dot{r}
\end{array}\right]=-I^{-1}\left[\begin{array}{l}
p \\
q \\
r
\end{array}\right] \times I\left[\begin{array}{l}
p \\
q \\
r
\end{array}\right]+I^{-1} \frac{1}{2} \rho V^{2} S\left[\begin{array}{lll}
b & 0 & 0 \\
0 & \bar{c} & 0 \\
0 & 0 & b
\end{array}\right]\left[\begin{array}{l}
C_{l} \\
C_{m} \\
C_{n}
\end{array}\right]
$$

With $I$ the moment of inertia, $b$ the wingspan and $\bar{c}$ the mean aerodynamic chord. Rewriting for the moment coefficients into the form of Eq. (7) by introducing a virtual control input $v_{\omega}$ gives:

$$
\begin{aligned}
{\left[\begin{array}{l}
C_{l} \\
C_{m} \\
C_{n}
\end{array}\right]_{r e q} } & =\frac{I}{\frac{1}{2} \rho V^{2} S}\left[\begin{array}{lll}
b & 0 & 0 \\
0 & \bar{c} & 0 \\
0 & 0 & b
\end{array}\right]^{-1}\left\{\left[\begin{array}{c}
v_{p} \\
v_{q} \\
v_{r}
\end{array}\right]+I^{-1}\left(\left[\begin{array}{c}
p \\
q \\
r
\end{array}\right] \times I\left[\begin{array}{c}
p \\
q \\
r
\end{array}\right]\right)\right\} \\
& =g_{\omega}^{-1}(\mathbf{x})\left[v_{\omega}-\mathbf{f}_{\omega}(\mathbf{x})\right]
\end{aligned}
$$

An outer sideslip control loop is implemented for the yaw channel using time scale separation with the yaw rate $r$ as control input. The sideslip angle can be written as:

$$
\beta=\arcsin \frac{v}{V}
$$

with $V$ the total true airspeed:

$$
V=\sqrt{u^{2}+v^{2}+w^{2}}
$$

Taking the time derivative of Eq. (15) gives:

$$
\dot{\beta}=\frac{\dot{v} V-v \dot{V}}{V \sqrt{u^{2}+w^{2}}}=\frac{\dot{v}}{\sqrt{u^{2}+w^{2}}}-\frac{v(u \dot{u}+v \dot{v}+w \dot{w})}{\left(u^{2}+v^{2}+w^{2}\right) \sqrt{u^{2}+w^{2}}}
$$

Substituting the following body axis equations for $\dot{u}, \dot{v}$ and $\dot{w}$ in Eq. (17):

$$
\begin{aligned}
& \dot{u}=A_{x}-g \sin \theta+r v-q w \\
& \dot{v}=A_{y}+g \sin \phi \sin \cos \theta-r u+p w \\
& \dot{w}=A_{z}+g \cos \theta \cos \phi+q u-p v
\end{aligned}
$$

and writing as an affine function of the yaw rate gives:

$$
\begin{aligned}
\dot{\beta}= & \frac{1}{\sqrt{u^{2}+v^{2}}}\left[\frac{-u v}{V^{2}}\left(A_{x}-g \sin \theta\right)+\left(1-\frac{v}{V^{2}}\right)\left(A_{y}+g \sin \phi \cos \theta\right)-\right. \\
& \left.\frac{v w}{V^{2}}\left(A_{z}+g \cos \phi \cos \theta\right)+w p_{r e f}\right]+\frac{-u}{\sqrt{u^{2}+w^{2}}} r_{r e f}
\end{aligned}
$$

Solving for the input $r$ by introducing the second virtual control input $v_{\beta}$ gives: 


$$
\begin{aligned}
r_{r e f}= & \left(\frac{-u}{\sqrt{u^{2}+w^{2}}}\right)^{-1}\left(v_{\beta}-\frac{1}{\sqrt{u^{2}+v^{2}}}\left[\frac{-u v}{V^{2}}\left(A_{x}-g \sin \theta\right)+\left(1-\frac{v}{V^{2}}\right)\right.\right. \\
& \left.\left.\times\left(A_{y}+g \sin \phi \cos \theta\right)-\frac{v w}{V^{2}}\left(A_{z}+g \cos \phi \cos \theta\right)+w p_{r e f}\right]\right) \\
= & g_{\beta}^{-1}(\mathbf{x})\left[v_{\beta}-f_{\beta}(\mathbf{x})\right]
\end{aligned}
$$

Parallel to the control structure in Figure 4 an airspeed NDI loop is added for thrust control. In the velocity axis reference frame the dynamics of the true airspeed is given by:

$$
\dot{V}=\left(A_{x}+\frac{T}{m}\right) \cos \alpha \cos \beta+A_{y} \sin \beta+A_{z} \sin \alpha \cos \beta
$$

Rewriting for the thrust lever input $T$ by introducing a third virtual control input $v_{V}$ gives:

$$
\begin{aligned}
T & =\left(\frac{1}{m} \cos \alpha \cos \beta\right)^{-1}\left[v_{V}-\left(A_{x} \cos \alpha \cos \beta+A_{y} \sin \beta+A_{z} \sin \alpha \cos \beta\right)\right] \\
& =g_{V}^{-1}(\mathbf{x})\left[v_{V}-f_{V}(\mathbf{x})\right]
\end{aligned}
$$

\section{Performance evaluation}

A high performance maneuver is used to evaluate the fault-tolerant controller. The maneuver is a combined pitch $q_{r e f}=8^{\circ} / \mathrm{s}$ and turn command $p_{\text {ref }}= \pm 15^{\circ} / \mathrm{s}$ at zero side-slip and constant airspeed $V_{\text {trim }}=83.5 \mathrm{~m} / \mathrm{s}$. The aircraft is trimmed at $\alpha_{\text {trim }}=10^{\circ}$. The maneuver is performed in three flight scenarios: 1 . The unfailed scenario (Fig. 5), 2. aileron hardover (Fig. 6) and 3. the structural damage scenario (Fig. 7). In both failure cases, the failure is activated after two seconds. The unfailed scenario serves as a comparison basis for the two failure cases.

The results for each scenario are presented through three sub figures. Subfigure (a) shows the response of the tracking quantities and aircraft states, subfigure (b) shows the control inputs and subfigure (c) shows the performance quantities which are the forgetting factor (Eq. (11)), the RLS innovation which is the error between the actual and the estimated moment coefficients:

$$
\varepsilon C_{M}(t)=C_{M}(t)-\hat{C}_{M}(t)
$$

and the control allocation error. The control allocation error is the error between the required moment coefficient delivered by the NDI controller and the actual moment coefficient delivered by the control allocator:

$$
\Delta C_{M}(t)=C_{M_{r e q}}(t)-C_{M}(t)
$$


The action of the identification algorithm is illustrated in Fig 8. This figure shows the onboard spline models for $C_{l}, C_{m}$ and $C_{n}$ at two time intervals during the maneuver. The models are parameterized as a function of the angle attack. The flight trajectories for the three scenarios are shown in Fig. 9. The performance assessment is based on the RMS values of the model errors, allocation errors and forgetting factor which are listed in Table 2.

\section{Nominal unfailed}

In the unfailed scenario tracking is satisfactory and minimal couplings exist in the three control channels as can be seen from Fig. 5a. From the forgetting factors in Fig. $5 \mathrm{c}$ and the updated models in Fig. 8 it can be observed that the controller is able to make small scale local modifications to the onboard spline model to reduce the errors within the control system. This property is a direct result of the high local approximation power of multivariate splines.

\section{Aileron hardover}

From Fig. 6 it can be observed that this failure does not represent a major challenge for the control system. The controller immediately compensates for the disturbing moment generated by the stuck aileron. There is no significant deterioration in the performance of the control system. There is only a slight increase in RLS innovations and control allocation errors as compared with the unfailed scenario, see Table 2, and the flight trajectories are nearly identical as can be seen from Fig. 9 .

\section{Structural damage}

This failure does also not represent a major challenge for the control system as can be seen from Fig. 7. Although the global aerodynamic properties of the aircraft have changed significantly the controller is still able to perform the requested maneuver. Again the control allocation and innovation RMS values have not increased significantly compared to the unfailed case. The higher value for RMS $\Delta C_{m}$ is also due to saturation of the elevator actuator as can be seen from Fig. 7b and Fig. 7c. From the innovations in Fig. $7 \mathrm{c}$ it can be seen that during excitation the controller detects the error and reduces it to zero mean quickly. The flight trajectory has changed significantly compared to the unfailed case which is to be expected since the aerodynamic properties have changed. 

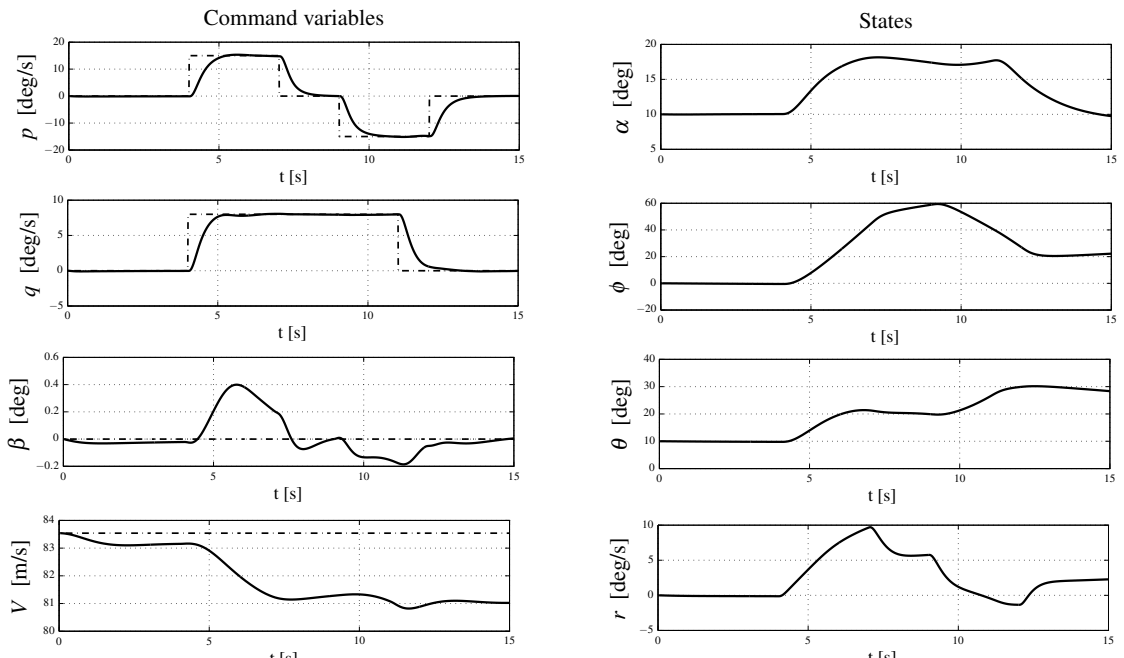

(a) Tracking response and states
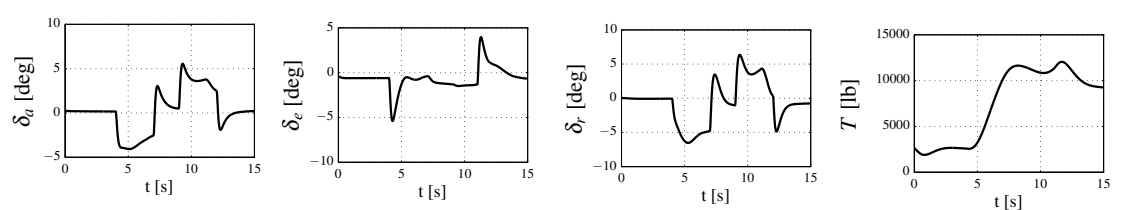

(b) Control deflections
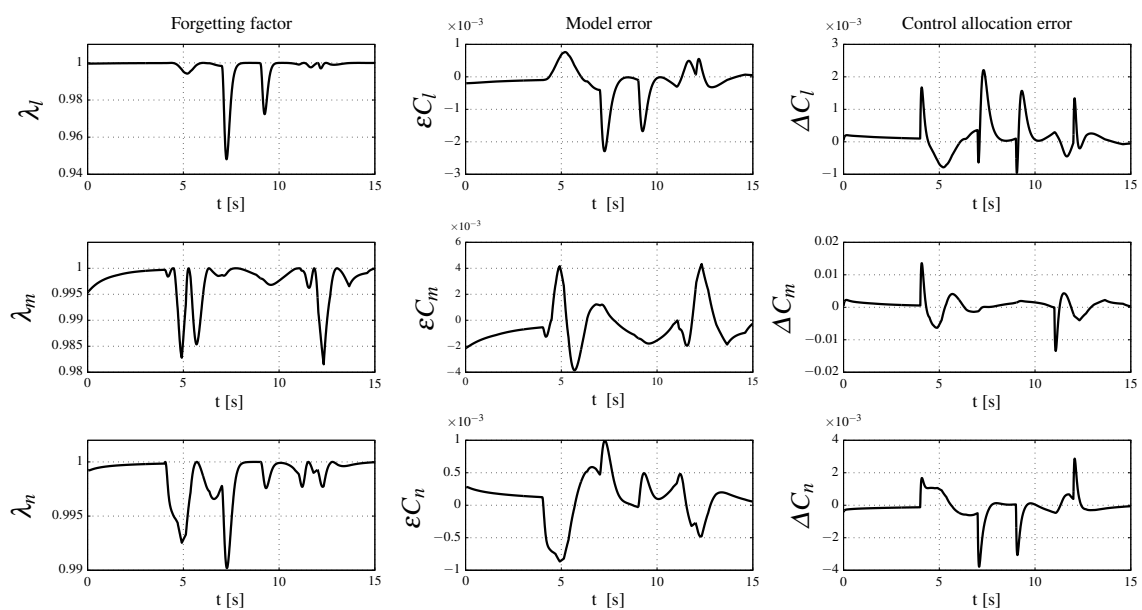

(c) Performance parameters: Forgetting factor, innovation and CA error

Fig. 5 Tracking quantities, states, control deflections and performance parameters for the unfailed scenario. 


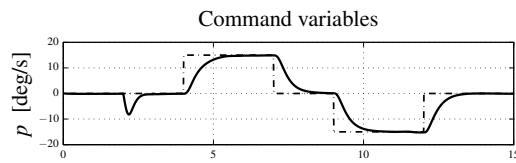

$\mathrm{t}$ [s]


$\mathrm{t}$ [s]

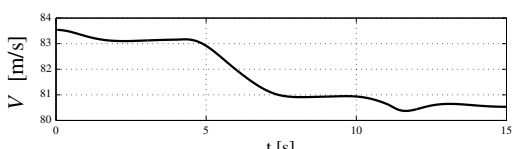

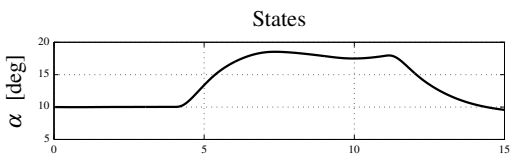

$\mathrm{t}$ [s]

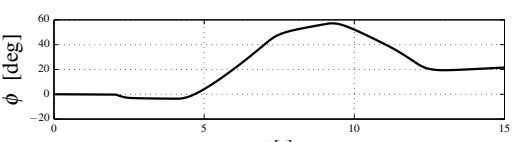

$\mathrm{t}[\mathrm{s}]$
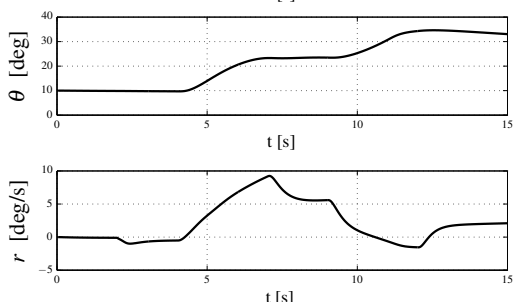

$\mathrm{t}[\mathrm{s}]$

(a) Tracking response and states
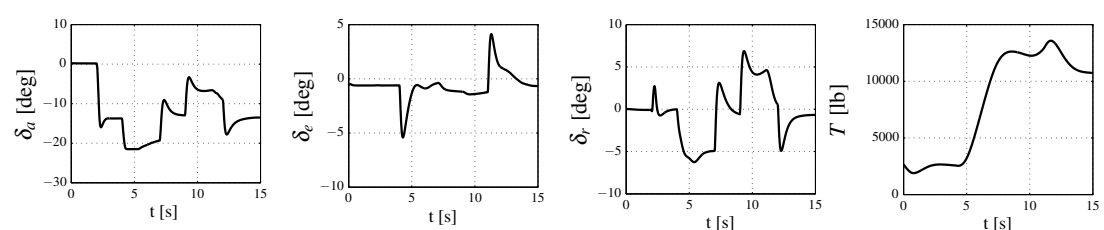

(b) Control deflections
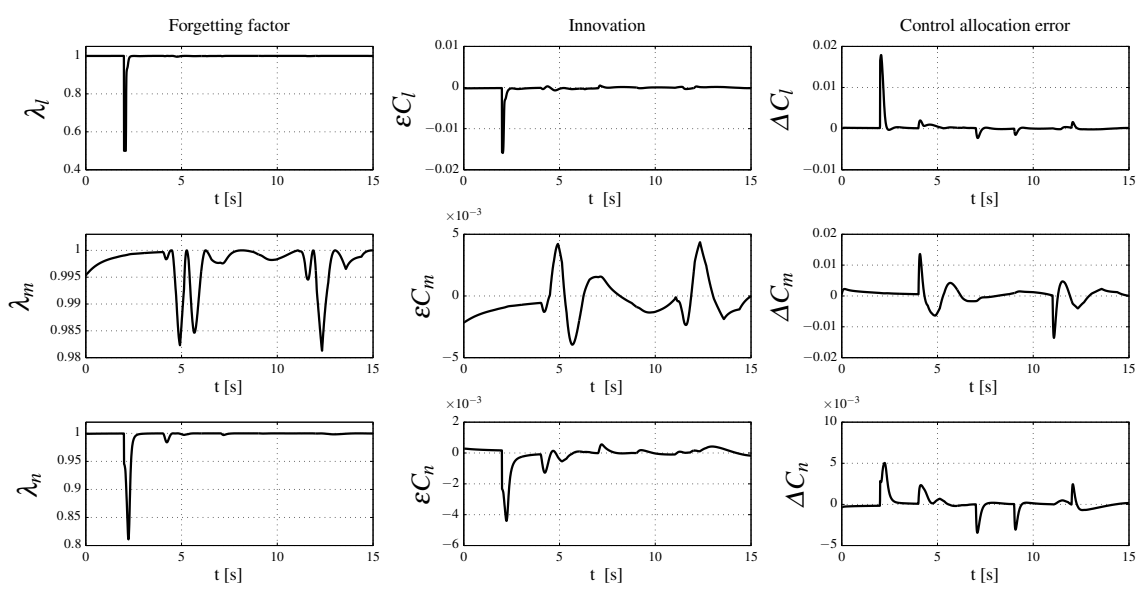

(c) Performance parameters: Forgetting factor, innovation and CA error

Fig. 6 Tracking quantities, states, control deflections and performance parameters for the aileron hard-over scenario. The failure is activated after 2 seconds. 

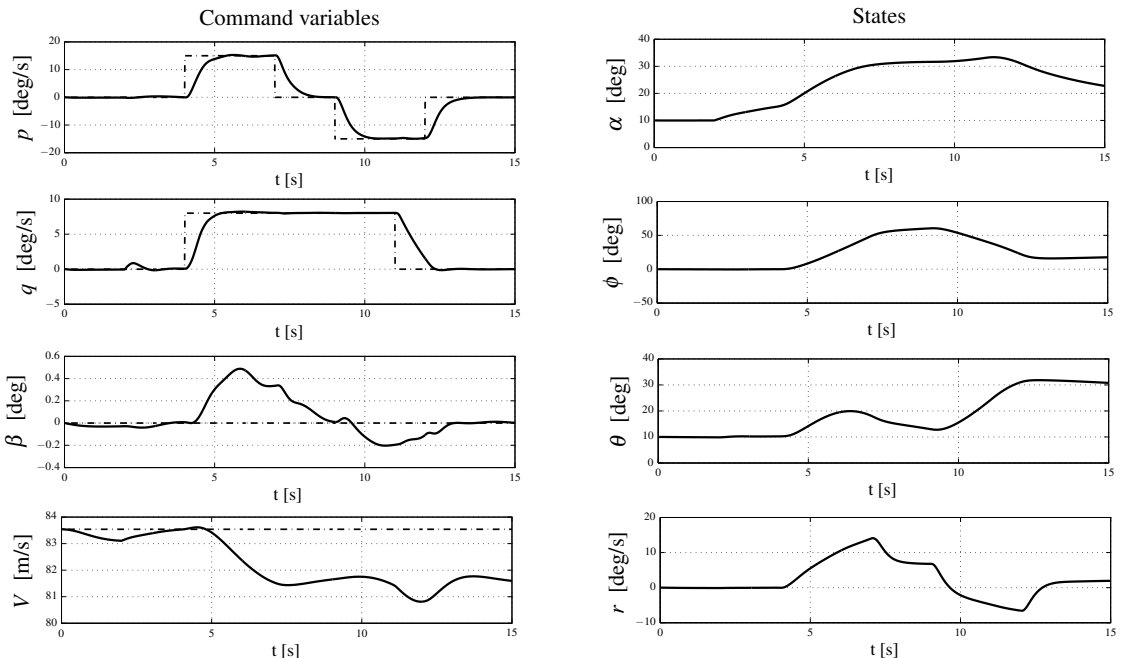

(a) Tracking response and states
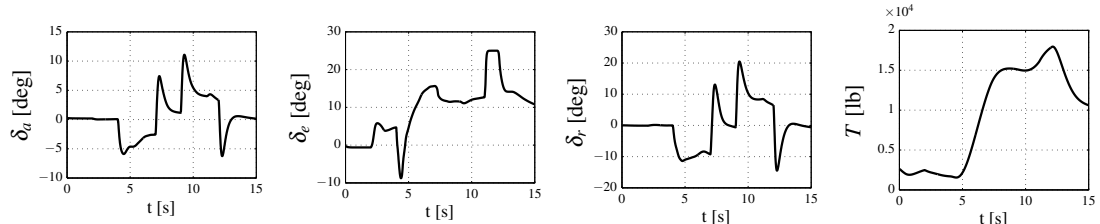

(b) Control deflections
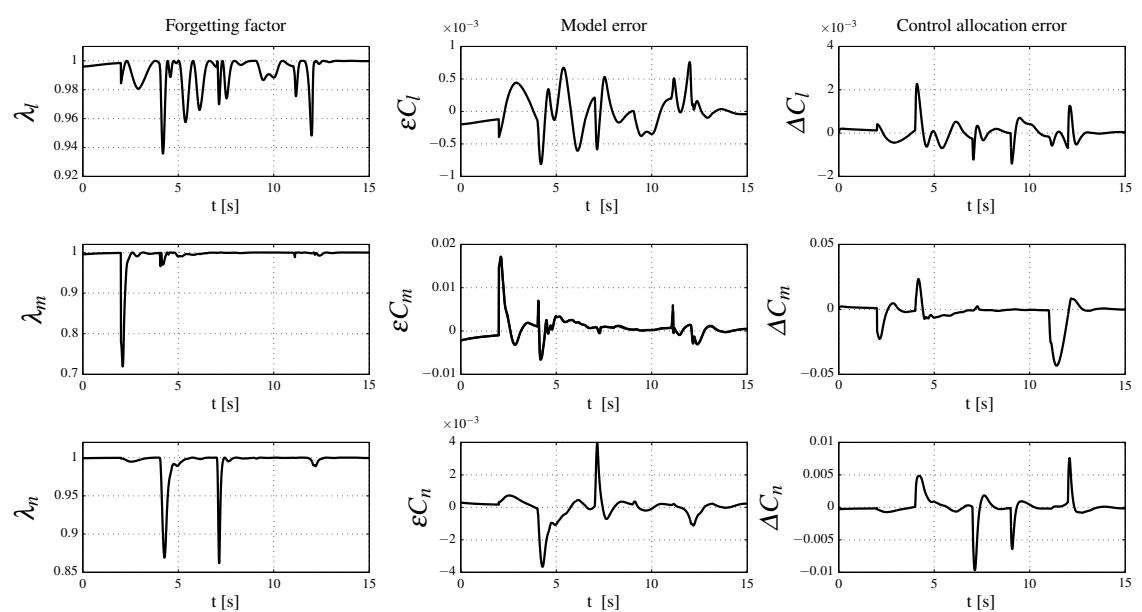

(c) Performance parameters: Forgetting factor, innovation and CA error

Fig. 7 Tracking quantities, states, control deflections and performance parameters for the structural failure scenario. The failure is activated after 2 seconds. 

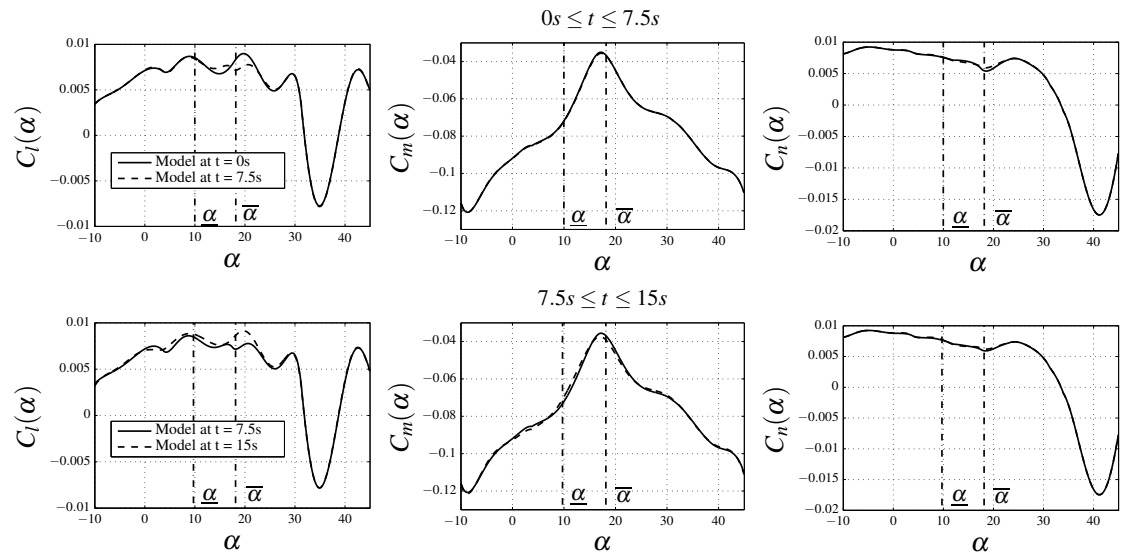

(a) Unfailed scenario
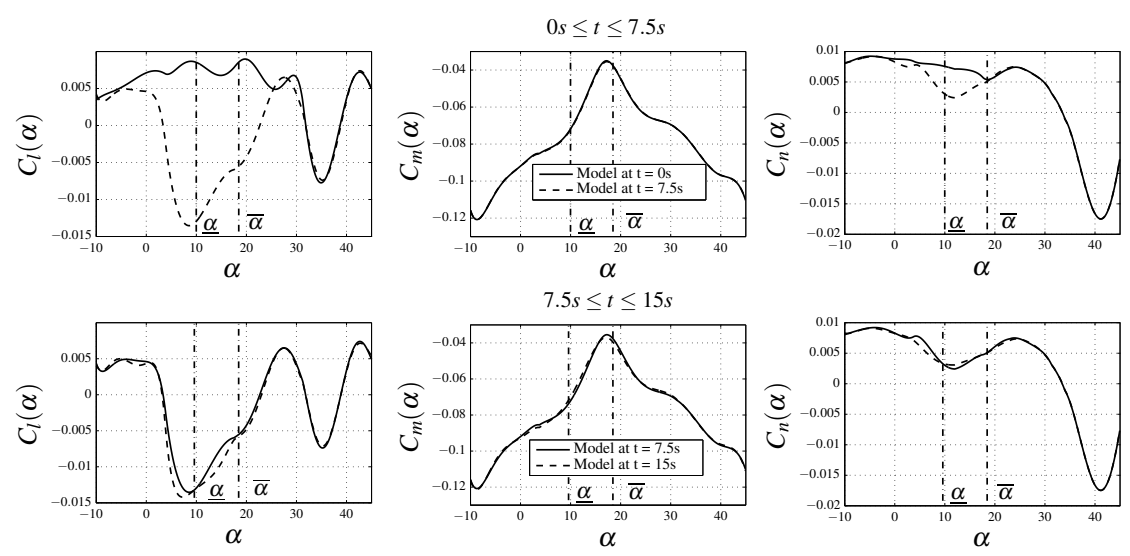

(b) Aileron hard-over scenario
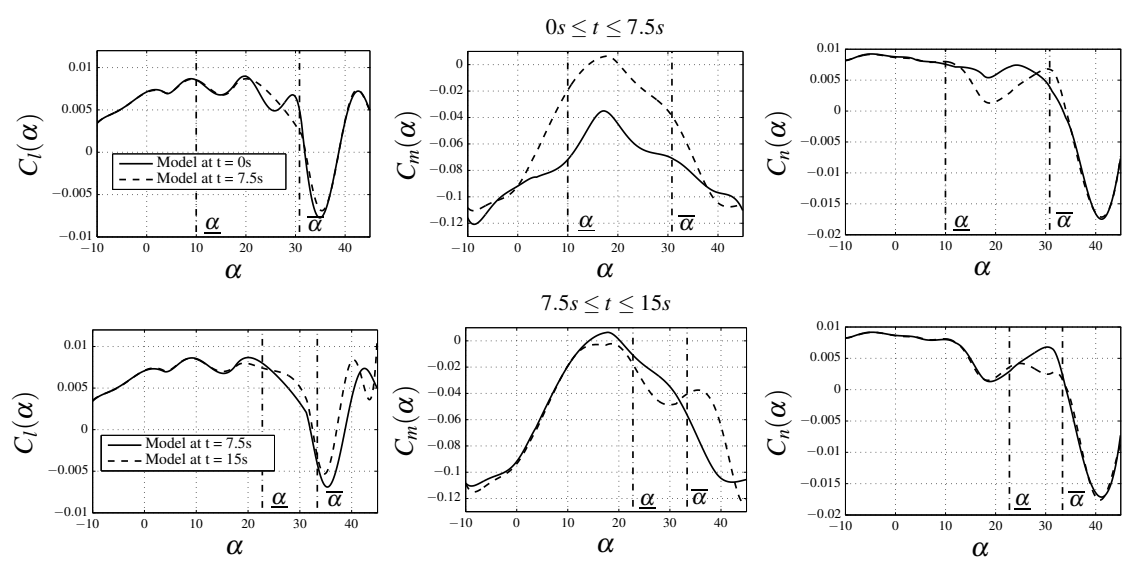

(c) Structural damage

Fig. 8 Updated models within two time intervals with: $\underline{\alpha} \leq \alpha \leq \bar{\alpha}$ the operating region within each interval. $\left(\beta=0^{o}, \tilde{p}=0.0049, \tilde{q}=0.0018, \tilde{r}=0.0049, \delta_{a}=-5^{o}\right.$, $\delta_{e}=5^{o}, \delta_{r}=-5^{o}, \delta_{l e f}=15^{\circ}$ ) 


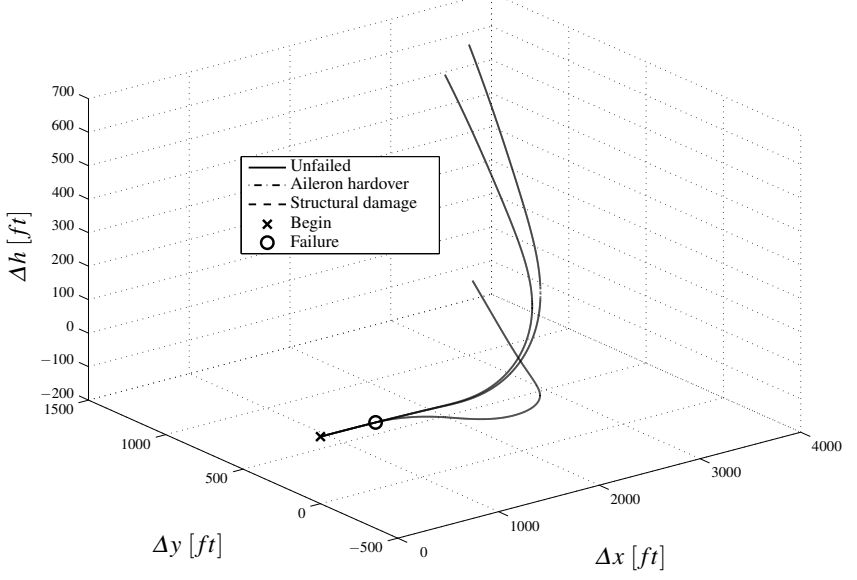

Fig. 9 Flight trajectories for the three scenarios

Table 2 Performance parameters for the three scenarios

\begin{tabular}{lccc}
\hline \hline & nominal & $\begin{array}{c}\text { aileron } \\
\text { hardover }\end{array}$ & $\begin{array}{c}\text { structural } \\
\text { damage }\end{array}$ \\
\hline RMS $\varepsilon C_{l}$ & 0.0002 & 0.0012 & 0.0003 \\
RMS $\varepsilon C_{m}$ & 0.0016 & 0.0016 & 0.0025 \\
RMS $\varepsilon C_{n}$ & 0.0004 & 0.0006 & 0.0007 \\
RMS $\Delta C_{l}$ & 0.0003 & 0.0017 & 0.0004 \\
RMS $\Delta C_{m}$ & 0.0026 & 0.0026 & 0.0094 \\
RMS $\Delta C_{n}$ & 0.0007 & 0.0009 & 0.0016 \\
Mean $\lambda_{l}$ & 0.9951 & 0.9942 & 0.9932 \\
Mean $\lambda_{m}$ & 0.9975 & 0.9975 & 0.9942 \\
Mean $\lambda_{n}$ & 0.9987 & 0.9963 & 0.9950 \\
\hline \hline
\end{tabular}

\section{5 conclusions}

This study shows that self-tuning adaptive control using multivariate splines is an effective and robust approach for fault-tolerant flight control. It is shown that the use of innovation based variable forgetting can circumvent the need for fault-detection and monitoring algorithms. Furthermore, the use of multivariate splines for real time model identification gives a robust adaptation of the internal model in case of a failure. 


\section{References}

1. Reiner, J., Balas, G. J., and Garrard, W. L., "Flight Control Design Using Robust Dynamic Inversion and Time-Scale Separation," Automatica, Vol. 32, No. 11, 1996, pp. 1493-1504.

2. Lane, S. H. and Stengel, R. F., "Flight Control Design Using Non-linear Inverse Dynamics," Automatica, Vol. 24, No. 4, 1988, pp. 471-483.

3. Walker, G. P. and Allen, D. A., "X-35b stovl Flight Control Law Design and Flying Qualities," Biennial International Powered Lift Conference and Exhibit, 2002.

4. Lombaerts, T. J. J., Van Oort, E. R., Chu, Q. P., Mulder, J. A., and Joosten, D. A., "Online Aerodynamic Model Structure Selection and Parameter Estimation for Fault-Tolerant Control." AIAA Journal of Guidance, Control, and Dynamics, Vol. 33, No. 3, 2010, pp. 707-723.

5. Lombaerts, T. J. J., Huisman, H. O., Chu, Q. P., Mulder, J. A., and Joosten, D. A., "Nonlinear Reconfiguring Flight Control Based on Online Physical Model Identification," AIAA Journal of Guidance, Control, and Dynamics, Vol. 32, 2009, pp. 727-748.

6. Lombaerts, T. J. J., Chu, Q. P., Mulder, J. A., and Joosten, D. A., "Modular Flight Control Reconfiguration Design and Simulation," Control Engineering Practice, Vol. 19, No. 6, 2011, pp. 540-554.

7. Calise, A. J., Lee, S., and Sharme, M., "Nonlinear Adaptive Flight Control Using Neural Networks," IEEE Control Systems, Vol. 18, 1998, pp. 14-25.

8. Kim, B. S. and Calise, A. J., "Nonlinear Flight Control Using Neural Networks," AIAA Journal of Guidance, Control and Dynamics, Vol. 20, No. 1, 1997, pp. 26-33.

9. Lai, M. J. and Schumaker, L. L., Spline Functions on Triangulations, Cambridge University Press, 2007.

10. de Visser, C. C., Chu, Q. P., and Mulder, J. A., "A New Approach to Linear Regression with Multivariate Splines," Automatica, Vol. 45, No. 12, 2009, pp. 2903-2909.

11. de Visser, C. C., Chu, Q. P., and Mulder, J. A., "Differential Constraints for Bounded Recursive Identification with Multivariate Splines," Automatica, Vol. 47, No. 9, 2011, pp. 2059-2066.

12. Tol, H. J., de Visser, C. C., van Kampen, E., and Chu, Q. P., "Nonlinear Multivariate SplineBased Control Allocation for High-Performance Aircraft," AIAA Journal of Guidance, Control, and Dynamics, Vol. 36, No. 6, 2014, pp. 1840-1862.

13. Nguyen, L. T., Ogburn, M. E., Gilbert, W. P., Kibler, K. S., Brown, P. W., and Deal, P. L., "Simulator Study of Stall/Post-Stall Characteristics of a Fighter Airplane with Relaxed Longitudinal Static Stability,” Tech. Rep. 1538, NASA, 1979.

14. Stevens, B. L. and Lewis, F. L., Aircraft Control and Simulation, John Wiley \& Sons, Hoboken, New Jersey, 2nd ed., 2003.

15. Choi, J. Y., Chwa, D., and Kim, M., "Adaptive Control for Feedback-Linearized Missiles with Uncertainties," IEEE Transactions on Aerospace and Electronic Systems, Vol. 36, No. 2, 2000, pp. 467-481.

16. de Visser, C. C., Chu, Q. P., and Mulder, J. A., "A Multidimensional Spline Based Global Nonlinear Aerodynamic Model for the Cessna Citation II," AIAA Atmospheric Flight Mechanics Conference, No. AIAA-2010-7950, 2010.

17. de Visser, C. C., Chu, Q. P., and Mulder, J. A., "Validating the Multidimensional Spline Based Global Aerodynamic Model for the Cessna Citation II," AIAA Atmospheric Flight Mechanics Conference, No. AIAA-2011-6356, 2011.

18. Fortescue, T. R., Kershenbaum, L. S., and Ydstie, B. E., "Implementation of Self-tuning Regulators with Variable Forgetting Factors," Automatica, Vol. 17, No. 6, 1981, pp. 831-835.

19. Wang, Q. and Stengel, R. F., "Robust Nonlinear Flight Control of a High-Performance Aircraft," IEEE Transactions on Systems Technology, Vol. 13, No. 1, 2005, pp. 15-26.

20. Lombaerts, T. J. J., Smali, M. H., Stroosma, O., Chu, Q. P., Mulder, J. A., and Joosten, D. A., "Piloted Simulator Evaluation Results of New Fault-Tolerant Flight Control Algorithm," AIAA Journal of Guidance, Navigation, and Control, Vol. 32, No. 6, 2009, pp. 1747-1765.

21. Sieberling, S., "Robust Flight control Using Incremental Nonlinear Dynamic Inverstion and Angular Acceleration Prediction," AIAA Journal of Guidance, Control, and Dynamics, Vol. 33, 2010, pp. 1732-1742. 